\title{
Solar wind test of the de Broglie-Proca's massive photon with Cluster multi-spacecraft data
}

\author{
Alessandro Spallicci* \\ Université d'Orléans, Observatoire des Sciences de l'Univers \\ E-mail: spallicciecnrs-orleans.fr
}

The exam of the literature on the large-scale astrophysical limits for the photon mass inspires a critical attitude and prompts to question whether these limits are nothing more than the outcome of idealized models. This view is confirmed by Goldhaber and Nieto [1] who state "Quoted photon-mass limits have at times been overly optimistic in the strengths of their characterizations. This is perhaps due to the temptation to assert too strongly something one 'knows' to be true. A look at the summary of the Particle Data Group [...] hints at this."

We use spacecraft data in the solar wind at $1 \mathrm{AU}$ to estimate the mass upper limit of the de Broglie-Proca's photon, by looking for deviations from the Ampère's law. We take advantage of the Cluster spacecraft which both allow the direct computation of $\nabla \times \vec{B}$ from simultaneous four-point measurements of the magnetic field and provide measurements of particle currents. We estimate the upper bound for the mass $m_{\gamma}$ to be $1.4 \times 10^{-49} \mathrm{~kg}$ without using any ad hoc model. Finally, we discuss how this limit can be lowered and compare with currently accepted values in the solar wind.

References

[1] Goldhaber and Nieto, Rev. Mod. Phys. 82, 939 (2010)

[2] A. Retinò, A. D. A. M. Spallicci and A. Vaivads, arXiv:1302.6168v2 [hep-ph] (2014).

Frontiers of Fundamental Physics 14 - FFP14,

15-18 July 2014

Aix Marseille University (AMU) Saint-Charles Campus, Marseille

${ }^{*}$ Speaker. 